\title{
On the Need for Democratic Principles to Ensure Meritocracy in a Time of Research Funding Cuts
}

\author{
Amparo Lasén \\ Universidad Complutense de Madrid \\ alasen@cps.ucm.es
}

Michèle Lamont's research on the academic evaluation system is a brilliant and much-needed contribution because it brings to light peer review procedures, challenging some assumptions about how scholars define and recognize excellence. She invites those of us involved in peer review and evaluation to be more reflexive about how we accomplish such a task, since it is the "holy grail" of academic life. Peer review represents the main procedure for allocating resources and positions. Yet in spite of its centrality, it is surrounded by opacity and secrecy most of the time; it is one of many academic tasks that are never taught, and we have to learn from our own experience. Since January 2011, I have been a member of the social sciences coordination team of the Agencia Nacional de Evaluación y Prospectiva (hereafter, ANEP), a public institution belonging to the State Secretary for Research, Development and Innovation, which is in charge of evaluating the main public research funding programs. Hence, as an ANEP member, I welcome the opportunity to participate in this symposium and to share some thoughts on this topic by drawing on my firsthand experience in academic evaluation, especially in the procedures for reaching agreement and defining the excellence of applicants and their proposals ${ }^{1}$.

My ANEP colleagues in the social sciences team and I share similar criteria for judging the quality of research proposals as those studied by Lamont: originality, feasibility, social and scientific interests. Similarly, there is no $a$ priori consensus about what is original, innovative, or interesting. This, too,

1. I would like to clarify that my current experience in this particular team is not necessarily similar to that of previous teams or current ANEP teams in other disciplines. Thus, my contribution exemplifies current evaluation processes in Spain but cannot be taken as a model of Spanish evaluative cultures. 
depends largely on evaluators' experiences and expertise. Therefore, my experience coincides with Lamont's account: reaching agreement and fair decisions after reading proposals and reviews resides in the deliberation process (where non-cognitive features, such as emotion work, play a key role) and in the ability to practice cognitive contextualization, namely, judging proposals in their own terms by using the epistemological (theoretical and methodological) styles that are most appropriate to the proposal. In the social sciences (and probably this applies to other disciplines as well), there are no standard criteria about how to define "what's interesting" (because there is no general and preliminary agreement about what topics are more interesting or socially relevant than others) or about how to balance originality and feasibility. These criteria emerge during the discussion. Applicants have to present and justify the interest, originality, and feasibility of their proposals, while reviewers have to judge whether the proposals are right by relying on their knowledge about the field, as well as by examining the applicants' research experience in order to evaluate their ability to complete the proposed project.

Though cognitive contextualization is a necessary condition for fair evaluation, it is still very common to find reviews - either in academic journals or in grants programs - where it is absent. By this, I am referring to reviewers who judge proposals according to their own epistemological style, as if they had in mind the proposal they would like to have written or wanted to read, instead of assessing the strengths and weaknesses of the proposal in question according to its particular content and style. On this point, I agree with Lamont. The deliberation process helps to put in practice such a cognitive contextualization (as opposed to just writing a review on our own or scoring proposals individually) when deploying and justifying our judgment in front of other scholars and reaching an agreement

In the ANEP programs I collaborate with, we tend to evaluate proposals and supervise reviewers' evaluations mostly within our own disciplines (sociology in my case). However, even in a single discipline, there is ample diversity of theories, methods, topics and interests; this requires using cognitive contextualization. Besides, in programs such as Ramón y Cajal and Juan de la Cierva ${ }^{2}$, panelists must evaluate proposals out of their personal expertise as well, and at the end of the process, they have to produce a single list ranking all candidates from all disciplines included in the social sciences ANEP area. ${ }^{3}$ The quality of the interactions during the meetings is crucial to reaching a fair outcome. Prior to the panel meeting, each of us evaluates a certain number of candidates; two panelists evaluate each candidate. We discuss via email the similarities and divergences in our reviews. But it is during the panel meeting when our notes

2. The Ramón y Cajal program seeks to hire talented researches by offering them five-year contracts and the possibility, at least until now, of a tenure-track position in the Spanish higher education system. The Juan de la Cierva program is a three-year postdoctoral position.

3. The disciplines are anthropology, sociology, political science, geography, journalism and communication, and library and information sciences. 
are put together; where the reviewers whose scoring of the same proposal is divergent can clarify their views; and where our practices of evaluating and scoring are brought together and adjusted according to the pragmatic fairness of deliberations described by Lamont in chapter 4. Thus, panelists' personal and collective interactive qualities are decisive for the success and fairness of the process (as well as the variety of approaches and areas covered by their expertise). This is one of the conditions and, at the same time, difficulties of a successful panel: to gather a maximum of diversity (regarding gender, approaches, epistemological styles, and geographical area of institutions) and to put in practice a robust cognitive contextualization, by bracketing their own interests and preferences, which is vital in order to recognize plural ways of identifying excellence and to carry out the debates and interactions enabling a fair evaluative outcome.

Although, as mentioned earlier, there are no a priori general criteria of evaluation of applicants' trajectory and of the quality of their teams' research, ${ }^{4}$ we must apply some standards established by public evaluation agencies. These criteria include the impact index of journals (hereafter, JCR), the quality of the book press where they have published, the competitive funding research programs they have joined (the most prestigious being the Plan Estatal de Investigación, at the national level, and The European Union Framework Program for Research and Innovation, at the European level) and the prestige of international universities and research centers where applicants have studied and worked. ${ }^{5}$ Since 2011, these shared standards are highlighted, as well, by the inclusion of scholars from European, specially British, and U.S. universities in the ANEP social science panels. Probably, the emphasis on internationalization (understood as publications in English in well-ranked journals and with important book publishers, funding from competitive research programs, such as those of the EU, research stays in prestigious universities abroad and participation in international scientific networks) is making our criteria closer to those described by Lamont.

Thus, beside the traditional hierarchical order of academia, a global hierarchy regarding institutions, journals and publishers emerges. In Spain, whereas older scholars at the top of academic hierarchies (at least in the fields I am more familiar with) did not need to be well-positioned in these global hierarchies and networks to be recognized as excellent, now those starting their academic careers have to prove their "internationalization," for instance, through a strong publishing record measured by the number of articles in JCR journals. This creates a paradoxical situation, for the CVs of most of evaluators do not include the kind of publications and international research experience expected from

4. In the case of proposals for the Plan Estatal de Investigación (State Plan of Research), projects are carried out by research teams and not by individual researchers.

5. In order to be taken into account as a merit, the period spent in a foreign academic center as a visiting scholar must be of at least six months. The merit, of course, is higher if applicants have obtained their doctorate or worked as research fellows in such centers. 
applicants whose excellence they have to evaluate. This does not necessarily mean that they will be unable to assess applicants and proposals with a higher level of internationalization and a better publishing record. It also does not entail that evaluators will not be able to practice cognitive contextualization in order to define excellence, as evaluators with strong international trajectory would do. Yet, it breaks with the implicit hierarchy between evaluators and applicants as described by a political scientist quoted by Lamont: "I felt like we were sitting on the top of a pyramid.....and were sorting between kind of $\mathrm{B}$, $\mathrm{B}+$, and A scholars, and we all thought we were As" (p. 1). My personal view is that losing this feeling of superiority towards applicants and proposals could produce a better affective situation for carrying out the review and putting cognitive contextualization into practice. Of course, it can elicit mixed feelings when we play the role of academic gatekeepers. But the acknowledgement of this paradox is not generalized and depends largely on how self-reflexive evaluators are. This is why many of them can still share the view of the political scientist quoted above regardless of their academic record.

When comparing the work of the panels described by Lamont with my experience at the ANEP, one of the main differences resides in how the institutional framework of funding programs and institutions contributes to producing and defining what excellence is. While the cases Lamont analyzes are fellowship and research grant programs funded by independent and private non-profit organizations, in Spain the main funding institutions are public, state agencies. In the current situation, due to drastic cuts in public spending and subsequent delays in issuing calls for proposals, the communication of results, transfers of funds, and modification of applications and reviewing of forms are also affecting the evaluation process profoundly. For instance, two weeks after finishing our evaluation for the Ramón y Cajal and Juan de la Cierva programs, the head of the social sciences commission informed us, in total dismay, that unlike previous years (when there was a list of candidates for each scientific area according to the different panels), this year the official guidelines asked us for a single general list, including all candidates from all scientific areas and stating who was eligible and who was in waiting positions. Such a list was not produced under a general evaluation framework, which would have been impossible since we do not share the same standards and epistemological styles across the so-called hard sciences, social sciences and the humanities. The reason for this decision made little sense to panellists and ANEP scientific teams, as well as the ANEP officials I work with, who had to produce as best as they could a single list from the ones provided by the different commissions. Funding cuts reduce the number of successful candidates, forcing us to remove excellent candidates from the eligible positions. Furthermore, hard-to-understand public decisions remind us that the peer review process is also shaped by political and institutional decisions totally out of scholars' reach. Unfortunately, in Spain in recent years, many of these decisions reveal a deep lack of responsibility and great ignorance about how scientific research works. 
Another example of organizational decisions that shape the process of identifying excellence is the design of the evaluation form filled by reviewers and panelists. These forms have different sections and scoring ranges. The Ramon y Cajal and Juan de la Cierva application forms changed in the last call. Whereas in 2011 there was one section for evaluating applicants' merits (publications, research experience, etc.) and another one for evaluating their research proposal, the latter has now been suppressed - applicants were not asked to describe the research project they would develop if they obtained the research contract. As a result of this change, excellence measured according to applicants' trajectory has taken over excellence as defined by their research project. (Cuts in research funding seem to be producing similar outcomes in other countries as well, in Canada (Gordon and Poulin 2013) and the United Kingdom (Rohn 2013).) This tendency can aggravate one of the pitfalls of the current peer review and academic evaluation process: the many versions of the "Matthew effect" or accumulated advantage, as Lamont describes it. Consequently, the reduction of the success rate due to research budget cuts risks giving more to those who have more now and have received more in the past.

Another shift in the application form this year (in line with changes due to funding cuts) concerns the division dedicated to assess applicants' merits in several sections: one for publications and participation in funded research projects and another just for their international experience in research projects and attainment of funding. Thus, applicants with an excellent publication record, but without much experience in international research projects or in obtaining funding from foreign agencies, cannot receive points from this section towards their final score, what clearly undermines their chances of being chosen. Applicants whose research does not require a large budget or who typically work on their own would also struggle to be recognized as excellent researchers according to this new type of evaluation form. As public investment in research funding decreases, the ability to obtain funding from other institutions is given more value in academic evaluation, and thus is transforming the understanding of what an excellent scholar is. If reviewers do not agree with such an understanding, they cannot override these new rules, since they have to follow the formal requirements of the evaluation process set by the funding institution.

The traditional scarcity of academic resources is increasing these days not only due to financial cuts, but also as a result of the globalization of some standards used to measure (or I should say "produce") excellence, such as the JCR impact factor, which leads everyone who wants to see their work recognized and to build a good publishing record to submit it to the same journals and presses. As success rates inevitably decrease, competition becomes tougher, and the time invested in being funded and published increases to the detriment of the time that scholars spend researching and disseminating research findings. This situation raises the issue of whether it is worth keeping this expensive evaluation process (measured in terms of money and time) when research funding is dwindling and when political decisions about research funding programs 
are erratic (Herbert et al. 2013). Some researchers are suggesting other ways of allocating research resources in a less costly and centralized fashion, such as giving a baseline grant to every qualified researcher working in academia who requests it (Gordon and Poulin 2013). This, in return, would be more inviting to those put off by time costs and reduced chances of success, and more democratic, with less paperwork before the allocation of funding and more accountability after the completion of research (Ibid.).

The diminishing success rate in obtaining research funding (due to financial cuts as well as the decreasing rate of papers published in top journals compared to the growing amount of papers submitted) is not related to a decrease of excellence within the academic community, since many excellent proposals and applicants are left without funding just because there is not enough money, and the publication of excellent papers is delayed and/or relegated to less well-ranked publications because top journals can only publish a limited number of them every year - not to mention other disadvantages, such as English not being most researchers' mother tongue, working on a geographical area less interesting for Anglo-Saxon journals or using non-English bibliographical references. If scholars' excellence is measured by their funded research and publication record, the increasing scarcity of academic resources can result in the production of scarce excellence. This risk is highlighted by the trend in emphasizing the excellence of trajectories instead of the excellence in research proposals and contributions.

Lamont cites some scholars who state that academia is a meritocracy, "intrinsically an elitist enterprise" and not a democracy (p. 217), an institution in which the rule of peer review and evaluation selects and promotes excellence and not the equal allocation of resources. However, as she clearly explains throughout How Professors Think, without establishing and sustaining democratic rules of deliberation, academic judgment is less likely to produce meritocracy than oligarchy and homophily. This is one of the reasons why it is important to have diversity in peer review panels. Diversity of topics, institutions, or gender is not a standard asked for in the evaluation requirements of the ANEP, and it is not something we tend to use in general as a criterion when producing lists of eligible proposals and candidates. We face the same situation described by Lamont, namely that merit and diversity do not respond to similar standards and that, in peer review procedures, merit prevails. But as Lamont finds, the numerous scholars accomplishing this task are not indifferent to diversity and think that a fair evaluation should produce a list of diverse eligible candidates or proposals. After completing our work and producing a list of successful candidates, it is not uncommon that we check how plural the list is; at least in our case of the current social sciences commission (I am not sure that it should be the case for everyone in our academic community), our satisfaction with the result runs parallel to the number of women, different disciplines, viewpoints, and institutions we have selected.

To conclude, I would like to quote my colleague at the Complutense University, Elena Casado, who says, half-joking, that academia very often seems to 
represent the worst of tradition, modernity, and postmodernity: the "feudal" hierarchies; the modernist nemesis of bureaucratic rationalization and the belief in a world split between experts and laypeople; and the postmodern tenet of "anything goes" and "do as you please," at least for those well-placed in the "old boys club." As a form of allocating scarce resources within academia, peer review serves as a gatekeeping mechanism for this particular institutionalized community, contributing therefore to these worst-of-three-worlds dynamic. But peer review can also prevent this dynamic. Since excellence is far from being universally self-evident, and identifying it is part of a deliberative process drawing on the ability to practice cognitive contextualization, then opening the black box of peer review as well as introducing democratic principles (such as transparency and diversity) are essential practices to guarantee research quality and innovation, that is, to produce a real meritocracy and not an empty and dusty set of practices serving a homogeneous oligarchy.

\section{Bibliographic References}

Gordon, Richard and Poulin, Bryan J. (2009). "Cost of the NSERC Science Grant Peer Review System Exceeds the Cost of Giving Every Qualified Researcher a Baseline Grant," Accountability in Research: Policies and Quality Assurance, 16(1), 13-40.

Herbert, Danielle L.; BarnetT, Adrian G. and Graves, Nicholas (2013). "Funding: Australia’s Grant System Wastes Time," Nature, 495, 314.

RoHn, Jenny (2013). "Show Me the Money: Is Grant Writing Taking over Science?," The Guardian <http://www.guardian.co.uk/science/occams-corner/2013/ apr/02/1>. Accessed on April 5, 2013. 